\title{
Lifting of an asymptotically inner flow for a separable $\mathrm{C}^{*}$-algebra
}

\author{
Akitaka Kishimoto \\ Department of Mathematics, Hokkaido University, Sapporo, Japan \\ kishi@math.sci.hokudai.ac.jp
}

\section{Introduction}

Before this symposium I had been pondering over approximately inner flows to see whether I could crack something with a new tool or two I might find in my toolbox. My approach was rather classical as usual; so the problem was to explore such flows in relation with invariant hereditary $\mathrm{C}^{*}$-subalgebras, extensions, tensor products, etc. To my disappointment I hardly got anything. Assuming nobody else has tried recently, the present knowledge on these flows does not seem to exceed much what is already presented in Bratteli-Robinson's book [2, 3] and Sakai's book [15]; notable results there are concerned with KMS states and representations in addition to a broad theory of unbounded derivations and generators and a theory in AF algebras.

My favorite (and only pertinent) result I had at that time was an existence result of approximately inner flows [8], which was obtained at the same time as the existence result of single automorphisms was in [12]. After the symposium I got a lifting theorem, which partly generalizes results by Pedersen, Olesen, and Elliott for universally weakly inner flows, referred to by Olesen at the conference (see $[13,4]$ ). But to prove this lifting theorem, I have to introduce a class of asymptotically inner flows in parallel with the case of single automorphisms; the result would say such a flow can be lifted from a quotient of a separable $\mathrm{C}^{*}$-algebra.

Without giving the definition precisely, I would say that all the known examples of approximately inner flows are actually asymptotically inner. In the next section I will give a few comments on this new notion and report on the existence result with some details. In section 3 I will then discuss the lifting theorem for flows. I will also add a similar result for automorphisms since I believe this has not been presented yet.

There was another type of flows I reported on in my talk, namely, the Rohlin flows, which are far from the asymptotically inner flows but could be more manageable by its strong property of cocycle vanishing (at least when 
the $\mathrm{C}^{*}$-algebra is a Kirchberg algebra $[5,6]$ ). I will not discuss it here (for interested readers see $[9,10,11])$.

I conclude this introduction by giving some basics on flows $[2,15]$ and the definition of asymptotically inner flows.

By a flow $\alpha$ on a $\mathrm{C}^{*}$-algebra $A$ we mean a homomorphism $\alpha: \mathbf{R} \rightarrow \operatorname{Aut}(A)$ such that $t \mapsto \alpha_{t}(x)$ is continuous for each $x \in A$, where $\operatorname{Aut}(A)$ is the automorphism group of $A$. When $\alpha$ is a flow, we denote by $\delta_{\alpha}$ the generator of $\alpha$, which is a closed derivation in $A$, i.e., $\delta_{\alpha}$ is a closed linear map defined on a dense $*$-subalgebra $D\left(\delta_{\alpha}\right)$ of $A$ into $A$ such that $\delta_{\alpha}(x)^{*}=\delta_{\alpha}\left(x^{*}\right)$ and

$$
\delta_{\alpha}(x y)=\delta_{\alpha}(x) y+x \delta_{\alpha}(y), \quad x, y \in D\left(\delta_{\alpha}\right) .
$$

Moreover $\delta_{\alpha}$ is well-behaved, or $\pm \delta_{\alpha}$ is dissipative. (But a well-behaved closed derivation need not be a generator.) Note that $D\left(\delta_{\alpha}\right)$ is a Banach $*$-algebra with the norm defined by embedding $D\left(\delta_{\alpha}\right)$ into $M_{2}(A)$ by

$$
x \mapsto\left(\begin{array}{cc}
x & \delta_{\alpha}(x) \\
0 & x
\end{array}\right) .
$$

Given $h \in A_{s a}, \delta_{\alpha}+$ ad $i h$ is again a generator, where ad $i h(x)=i(h x-$ $x h), x \in A$. We denote by $\alpha^{(h)}$ the flow generated by $\delta_{\alpha}+\operatorname{ad} i h$. We call $\alpha^{(h)}$ an inner perturbation of $\alpha$. More generally, if $u$ is an $\alpha$-cocycle, i.e., $u: \mathbf{R} \rightarrow \mathcal{U}(A)$ is continuous such that $u_{s} \alpha_{s}\left(u_{t}\right)=u_{s+t}, s, t \in \mathbf{R}$, then $t \mapsto$ Ad $u_{t} \alpha_{t}$ is a flow, called a cocycle perturbation of $\alpha$. Note that an inner perturbation is a cocycle perturbation; $\alpha^{(h)}$ is obtained as $\operatorname{Ad} u^{(h)} \alpha$, where $u=u^{(h)}$ is the (differentiable) $\alpha$-cocycle defined by $d u_{t} / d t=u_{t} \alpha_{t}(i h)$ and $u_{0}=1$. In general a cocycle perturbation of $\alpha$ is given as $t \mapsto \operatorname{Ad} v \alpha_{t}^{(h)} \operatorname{Ad} v^{*}=$ $\operatorname{Ad}\left(v u^{(h)} \alpha_{t}\left(v^{*}\right)\right) \alpha_{t}$ for some $v \in \mathcal{U}(A)$ and $h \in A_{s a}$.

We will use the following result below (see $[2,15])$.

Proposition 1. Let $\alpha$ be a flow on a $C^{*}$-algebra $A$ and let $\left(h_{n}\right)$ be a sequence in $A_{s a}$. Then the following conditions are equivalent.

1. $\lim _{n \rightarrow \infty} \max _{|t| \leq 1}\left\|\alpha_{t}(x)-\operatorname{Ad} e^{i t h_{n}}(x)\right\|=0, x \in A$.

2. $\delta_{\alpha}=\lim _{n \rightarrow \infty}$ ad $i h_{n}$ in the graph sense, i.e., for any $x \in D\left(\delta_{\alpha}\right)$ there is a sequence $\left(x_{n}\right)$ in $A$ such that $\lim _{n \rightarrow \infty}\left\|x-x_{n}\right\|=0$ and $\lim _{n \rightarrow \infty} \| \delta_{\alpha}(x)-$ ad $i h_{n}\left(x_{n}\right) \|=0$.

The flow $\alpha$ as in the above proposition is called an approximately inner flow. Let us define asymptotically inner flows in the same way as we do asymptotically inner automorphisms for approximately inner automorphisms.

Definition 2. A flow $\alpha$ on a $C^{*}$-algebra $A$ is said to be asymptotically inner if there is a continuous function $h$ of $\mathbf{R}_{+}$into $A_{\text {sa }}$ such that

$$
\lim _{s \rightarrow \infty} \max _{|t| \leq 1}\left\|\alpha_{t}(x)-\operatorname{Ad} e^{i t h(s)}(x)\right\|=0
$$

for any $x \in A$. In this case we say that $\mathrm{Ad} e^{i t h(s)}$ converges to $\alpha_{t}$ as $s \rightarrow \infty$ or $\alpha_{t}$ is the limit of $\operatorname{Ad} e^{i t h(s)}$ as $s \rightarrow \infty$. 
It is obvious that asymptotical innerness implies approximate innerness. We know, for single automorphisms, that the class of asymptotically inner automorphisms is smaller than the class of approximately inner automorphisms in general (see $[5,6])$.

The following is an easy corollary of what is shown in $[4,13]$ and gives a class of asymptotically inner flows.

Proposition 3. Let $A$ be a separable $C^{*}$-algebra and let $\alpha$ be a flow on $A$. If $\alpha$ is universally weakly inner (i.e., there is a weak $k^{*}$-continuous unitary flow $u$ in $A^{* *}$ such that $\left.\alpha_{t}(x)=\operatorname{Ad} u_{t}(x), x \in A\right)$, then it is asymptotically inner.

If $A$ is unital and simple, then a universally weakly inner flow is just uniformly continuous and hence is inner.

\section{Asymptotically inner flows}

With the two similar properties for flows at hand, I suppose we must say something about them. What I have to confess is that I do not know whether or not the notion of asymptotical innerness is strictly stronger than the one of approximate innerness. But a naive expectation would fail. For example, if $\alpha$ is a flow on $A$ and $\left(h_{n}\right)$ is a sequence in $A_{s a}$ such that $\alpha_{t}$ is the limit of $\operatorname{Ad} e^{i t h_{n}}$, we may expect, defining $h: \mathbf{R}_{+} \rightarrow A_{s a}$ by $h(s)=(n-s) h_{n-1}+(s-n+1) h_{n}, s \in$ $[n-1, n]$, that $\alpha_{t}$ is the limit of $\operatorname{Ad} e^{i t h(s)}$ as $s \rightarrow \infty$, which is not the case in general as shown by the following simple example.

Example 4. Let $A$ be a unital simple AF algebra and let $\left(A_{n}\right)$ be an increasing sequence of finite-dimensional $\mathrm{C}^{*}$-subalgebras of $A$ such that $\bigcup_{n} A_{n}$ is dense in $A$. Let $h, a \in\left(A_{1}\right)_{s a}$ be such that $[h, a] \neq 0$. Let $x_{n}, y_{n} \in\left(A \cap A_{n}^{\prime}\right)_{s a}$ be such that $\left\|y_{n}\right\|=1$ and $\left\|\left[x_{n}, y_{n}\right]\right\| \rightarrow \infty$ (and so $\left.\left\|x_{n}\right\| \rightarrow \infty\right)$. Let $\epsilon_{n}=\left\|\left[x_{n}, y_{n}\right]\right\|^{-1}$ and define

$$
h_{2 n-1}=e^{i \epsilon_{n} h y_{n}} x_{n} e^{-i \epsilon_{n} h y_{n}} \approx x_{n}+i \epsilon_{n} h\left[y_{n}, x_{n}\right]
$$

(with an error of order $\epsilon_{n}$ ) and $h_{2 n}=-x_{n}$.

Then $\operatorname{Ad} e^{i t h_{n}}$ converges to the trivial flow id since $e^{i \epsilon_{n} h y_{n}}$ converges to 1 and $\operatorname{Ad} e^{i t x_{n}}$ converges to id. Let

$$
k_{n}=\frac{1}{2}\left(h_{2 n-1}+h_{2 n}\right) \approx \frac{1}{2} i \epsilon_{n} h\left[y_{n}, x_{n}\right] .
$$

It then follows that $\left(k_{n}\right)$ is a bounded sequence and that $\left(\operatorname{ad} i k_{n}\right)$ does not converge since $\operatorname{ad} i k_{n}(a) \approx i[h, a] \epsilon_{n}\left[y_{n}, x_{n}\right]$. This implies that $\operatorname{Ad} e^{i t k_{n}}$ does not converge.

We often encounter the situation given in the following proposition: 
Proposition 5. Let $\alpha$ be a flow on a $C^{*}$-algebra A. Suppose that there is a sequence $\left(h_{n}\right)$ in $A_{s a}$ and a $*$-subalgebra $D$ of $A$ such that $D \subset D\left(\delta_{\alpha}\right), D$ is a core for $\delta_{\alpha}$, and

$$
\delta_{\alpha}(x)=\lim _{n \rightarrow \infty} \operatorname{ad} i h_{n}(x), \quad x \in D .
$$

Then $\alpha$ is asymptotically inner.

Proof. In this situation we define a continuous function $h: \mathbf{R}_{+} \rightarrow A_{s a}$ by

$$
h(s)=(n-s) h_{n-1}+(s-n+1) h_{n}, \quad s \in[n-1, n]
$$

with $h_{0}=0$. Then it follows that $\delta_{\alpha}(x)=\lim _{s \rightarrow \infty}$ ad $i h(s)(x), x \in D$ and that $\delta_{\alpha}$ is the graph limit of ad $i h(s)$ as $s \rightarrow \infty$. From this the conclusion follows.

The core condition for $D$ above may not be possible to prove. Another situation we may encounter is as follow:

Proposition 6. Let $\alpha$ be a flow on a $C^{*}$-algebra A. Suppose that there is a sequence $\left(h_{n}\right)$ in $A_{\text {sa }}$ and a dense $*$-subalgebra $D$ of $A$ such that $\delta_{\alpha}$ is the graph limit of $\left(\operatorname{ad} i h_{n}\right), h_{n} \in D \subset D\left(\delta_{\alpha}\right)$,

$$
\delta_{\alpha}(x)=\lim _{n \rightarrow \infty} \operatorname{ad} i h_{n}(x), \quad x \in D,
$$

and $\left(\left\|\delta_{\alpha}\left(h_{n}\right)\right\|\right)$ is bounded. Then $\alpha$ is asymptotically inner.

Proof. We define a continuous function $h: \mathbf{R}_{+} \rightarrow A_{s a}$ by linearly interpolating $n \mapsto h_{n}$ as in the previous proof. Then it follows that for any increasing sequence $\left(s_{n}\right)$ in $\mathbf{R}_{+}$such that $s_{n} \rightarrow \infty$, the sequence $\left(h\left(s_{n}\right)\right)$ satisfies the same conditions as $\left(h_{n}\right)$ does, except that $\delta_{\alpha}$ is the graph limit of $\left(\operatorname{ad} i h\left(s_{n}\right)\right)$.

Suppose that $\left(s_{n}\right)$ includes $\mathbf{N}$ as a subsequence. Let $\delta$ be the graph limit of $\left(\operatorname{ad} i h\left(s_{n}\right)\right)$. Then $\delta$ is a restriction of $\delta_{\alpha}$ such that $D(\delta) \supset D$. It follows from 3.1 of [1] that $\delta$ is a generator, i.e., $\delta=\delta_{\alpha}$. Thus we can conclude that $\alpha$ is asymptotically inner.

A flow $\alpha$ is an asymptotically inner perturbation of a flow $\beta$ if there is a continuous function $h: \mathbf{R}_{+} \rightarrow A_{s a}$ such that $\operatorname{Ad} \beta_{t}^{(h(s))}$ converges to $\alpha_{t}$, i.e.,

$$
\lim _{s \rightarrow \infty} \max _{|t| \leq 1}\left\|\alpha_{t}(x)-\beta_{t}^{(h(s))}(x)\right\|=0
$$

for any $x \in A$.

With this definition, an asymptotically inner flow is an asymptotically inner perturbation of the trivial flow id. Then there arises a natural problem: If $\alpha$ is an asymptotically inner flow, is the trivial flow id an asymptotically inner perturbation of $\alpha$ ? Although this looks quite plausible, I am embarrassed to say that I do not know the answer. But again a naive expectation would fail: If $\alpha_{t}$ is the limit of $\operatorname{Ad} e^{i t h(s)}$ as $s \rightarrow \infty, \alpha^{(-h(s))}$ need not converge to id as $s \rightarrow \infty$ as shown as follows: 
Example 7. Let $A$ be a unital simple AF algebra and let $\alpha$ be a flow on $A$ such that there is an increasing sequence $\left(A_{n}\right)$ of unital finite-dimensional $\mathrm{C}^{*}$-subalgebras of $A$ such that $A=\overline{\bigcup_{n} A_{n}}$ and $\bigcup_{n} A_{n} \subset D\left(\delta_{\alpha}\right)$. Suppose that $\bigcup_{n} A_{n}$ is a core for $\delta_{\alpha}$ and $\delta_{\alpha}$ is unbounded. Then there is a continuous function $h: \mathbf{R}_{+} \rightarrow A_{s a}$ such that $\delta_{\alpha}$ is the graph limit of ad $i h(s)$ as $s \rightarrow \infty$ but $\delta_{\alpha}-\operatorname{ad} i h(s)$ does not converge to zero in the graph sense as $s \rightarrow \infty$.

In the above situation there is a $k_{n} \in A_{s a}$ such that $\delta_{\alpha}\left|A_{n}=\operatorname{ad} i k_{n}\right| A_{n}$. Then since $\bigcup_{n} A_{n}$ is a core for $\delta_{\alpha}$, we get that ad $i k_{n}$ converges to $\delta_{\alpha}$ as $n \rightarrow \infty$ in the graph sense. Moreover, since $\delta_{\alpha}-\operatorname{ad} i k_{n}$ converges to zero on each element of $\bigcup_{n} A_{n}$, we get that $\delta_{\alpha}-$ ad $i k_{n}$ converges to zero in the graph sense. By passing to a subsequence of $\left(A_{n}\right)$ and by giving a small inner perturbation to $\delta_{\alpha}$, we may suppose that $\delta_{\alpha}\left(A_{n}\right) \subset A_{n+1}$.

Assume that $A_{1}$ is not commutative and fix $a, x \in\left(A_{1}\right)_{s a}$ such that $\|a\|=1$ and $[a, x] \neq 0$. We find a sequence $\left(b_{n}\right)$ in $A_{s a}$ and a sequence $\left(\ell_{n}\right)$ in $\mathbf{N}$ such that $b_{n} \in A_{n} \cap A_{\ell_{n}}^{\prime},\left\|b_{n}\right\|=1,\left\|\delta_{\alpha}\left(b_{n}\right)\right\| \rightarrow \infty, n \geq \ell_{n}$, and $\ell_{n} \rightarrow \infty$. We set $u_{n}=e^{i \epsilon_{n} a b_{n}}$, where $\epsilon_{n}=\max \left\{1,\left\|\delta_{\alpha}\left(b_{n}\right)\right\|\right\}^{-1}$. We define $h_{n}=u_{n} k_{n+1} u_{n}^{*}$. Since $u_{n} \rightarrow 1$ and

$$
\operatorname{Ad} e^{i t h_{n}}(y)=u_{n} \operatorname{Ad} e^{i t k_{n+1}}\left(u_{n}^{*} y u_{n}\right) u_{n}^{*},
$$

Ad $e^{i t h_{n}}$ converges to $\alpha_{t}$ as $n \rightarrow \infty$ or ad $i h_{n}$ converges to $\delta_{\alpha}$ in the graph sense.

We can interpolate $\left(h_{n}\right)$, i.e., we have a continuous function $h: \mathbf{R}_{+} \rightarrow A_{s a}$ such that $h(n)=h_{n}$ (and perhaps taking on $k_{n+1}$ after and before $n$ ) and ad $i h(s)$ converges to $\delta_{\alpha}$ in the graph sense.

We assert that $\delta_{\alpha}-$ ad $i h(s)$ does not converge to zero (in the graph sense). For this purpose it suffices to show that $\delta_{\alpha}-$ ad $i h_{n}$ does not converge to zero.

Suppose that $\delta_{\alpha}-$ ad $i h_{n}$ converge to zero, which implies that $\operatorname{Ad} u_{n}^{*}\left(\delta_{\alpha}-\right.$ ad $\left.i h_{n}\right) \operatorname{Ad} u_{n}=\delta_{\alpha}+\operatorname{ad} u_{n}^{*} \delta_{\alpha}\left(u_{n}\right)-\operatorname{ad} i k_{n+1}$ also converge to zero.

Since $\left\|\delta_{\alpha}\left(u_{n}\right)-i \epsilon_{n} a \delta_{\alpha}\left(b_{n}\right)\right\| \rightarrow 0$, we get that

$$
\delta_{\alpha}-\operatorname{ad} i k_{n+1}+\operatorname{ad} i \epsilon_{n} a \delta_{\alpha}\left(b_{n}\right) \rightarrow 0 .
$$

For the $x \in A_{1}$ we have chosen before, we get a sequence $\left(x_{n}\right)$ in $D\left(\delta_{\alpha}\right)$ such that $\left\|x-x_{n}\right\| \rightarrow 0$ and

$$
\delta_{\alpha}\left(x_{n}\right)-\operatorname{ad} i k_{n+1}\left(x_{n}\right)+i \epsilon_{n}\left[a, x_{n}\right] \delta_{\alpha}\left(b_{n}\right) \rightarrow 0,
$$

where we have used that $\delta_{\alpha}\left(b_{n}\right) \in A_{n+1} \cap A_{\ell_{n}-1}^{\prime}$. We will show that this is absurd.

Let $\beta$ denote the flow generated by $\delta_{\alpha}-$ ad $i k_{n+1}$. Then $\beta_{t} \mid A_{n+1}=$ id. Note that $[a, x] \in A_{1}$ and $\delta_{\alpha}\left(b_{n}\right) \in A_{n+1} \cap A_{\ell_{n}-1}^{\prime}$ as asserted above. Since $A$ is simple, we get that $\left\|[a, x] \delta_{\alpha}\left(b_{n}\right)\right\|=\|[a, x]\| \cdot\left\|\delta_{\alpha}\left(b_{n}\right)\right\| \neq 0$ for all large $n$. Let $\phi_{n}$ be a state of $A_{n+1}$ such that $\left|\phi_{n}\left(\epsilon_{n}[a, x] \delta_{\alpha}\left(b_{n}\right)\right)\right|=\|[a, x]\|$ for such $n$. Let $\bar{\phi}_{n}$ be an extension of $\phi_{n}$ to a state of $A$ and let $\psi_{n}$ be an average of $\bar{\phi}_{n} \beta_{t}$ over $t \in \mathbf{R}$. Then, since $\psi_{n} \circ\left(\delta_{\alpha}-\operatorname{ad} i k_{n+1}\right)=0$ and $\psi_{n} \mid A_{n+1}=\phi_{n}$, we get that 


$$
\begin{aligned}
& \left|\psi_{n}\left(\delta_{\alpha}\left(x_{n}\right)-\operatorname{ad} i k_{n+1}\left(x_{n}\right)+\operatorname{ad} i \epsilon_{n}\left[a, x_{n}\right] \delta_{\alpha}\left(b_{n}\right)\right)\right| \\
\rightarrow & \lim _{n \rightarrow \infty}\left|\psi_{n}\left(i \epsilon_{n}[a, x] \delta_{\alpha}\left(b_{n}\right)\right)\right|=\|[a, x]\|,
\end{aligned}
$$

which is a contradiction.

We note the following result.

Proposition 8. Suppose that the $C^{*}$-algebra is unital. The following statements hold.

1. A cocycle perturbation of an asymptotically inner flow is asymptotically inner.

2. A asymptotically inner perturbation of an asymptotically inner flow is asymptotically inner.

Proof. Let $\alpha$ be an asymptotically inner flow on $A$ and let $h: \mathbf{R}_{+} \rightarrow A_{s a}$ be such that $\alpha_{t}$ is the limit of $\operatorname{Ad} e^{i t h(s)}$ as $s \rightarrow \infty$.

If $b \in A_{s a}$, let $u_{t}$ denote the $\alpha$-cocyle such that $d u_{t} /\left.d t\right|_{t=0}=i b$ and let $u_{t}^{(s)}$ denote the Ad $e^{i t h(s)}$-cocycle such that $d u_{t}^{(s)} /\left.d t\right|_{t=0}=i b$. Then, by the explicit series expansions of $u_{t}$ and $u_{t}^{(s)}$, we have that

$$
\lim _{s \rightarrow \infty} \max _{|t| \leq 1}\left\|u_{t}-u_{t}^{(s)}\right\|=0 .
$$

Thus it follows that $\alpha_{t}^{(b)}=\operatorname{Ad} u_{t} \alpha_{t}$ is obtained as the limit of $\operatorname{Ad} e^{i t(h(s)+b)}=$ $\operatorname{Ad} u_{t}^{(s)} \operatorname{Ad} e^{i t h(s)}$.

If $z \in A$ is a unitary, there is a unitary $w \in D\left(\delta_{\alpha}\right)$ such that $\|z-w\|<2$. We express $z w^{*}=e^{i h}$ for some $h \in A_{s a}$ and find a continuous function $k: \mathbf{R}_{+} \rightarrow D\left(\delta_{\alpha}\right) \cap A_{s a}$ such that $k(0)=0$ and $\lim _{s \rightarrow \infty}\|k(s)-h\|=0$ (where we assume that $s \mapsto \delta_{\alpha}(k(s))$ is continuous as well as $\left.s \mapsto k(s)\right)$. Namely, by taking $s \mapsto e^{i k(s)} w$, we find a continuous function $v: \mathbf{R}_{+} \rightarrow D\left(\delta_{\alpha}\right) \cap \mathcal{U}(A)$ such that $\lim _{s \rightarrow \infty}\|z-v(s)\|=0$. Define a continuous function $h \rightarrow A_{s a}$ by $h(s)=-i v(s) \delta_{\alpha}\left(v(s)^{*}\right)$. Then $\alpha_{t}^{(h(s))}$ converges to $\operatorname{Ad} z \alpha_{t} \operatorname{Ad} z^{*}$ (although $\|h(s)\| \rightarrow \infty$ if $z \notin D\left(\delta_{\alpha}\right)$ ). This completes the proof of (1) (by using (2) below) since any $\alpha$-cocycle is given as $z u_{t} \alpha_{t}\left(z^{*}\right)$ with $u_{t}$ differentiable.

Furthermore if $k: \mathbf{R}_{+} \rightarrow A_{s a}$ is continuous and a flow $\beta$ is obtained as the limit of $\alpha^{(k(s))}$ with $\alpha$ as above, one can easily see that for any $x \in A$, the continuous function $[-1,1] \ni t \mapsto \beta_{t}(x)$ can be approximated by $[-1,1] \ni$ $t \mapsto \alpha_{t}^{(k(s))}(x)$ for large $s \in \mathbf{R}_{+}$and then approximated by $[-1,1] \ni t \mapsto$ Ad $e^{i t(h(\sigma)+k(s))}(x)$ (from the first part of the proof of (1)), where $\sigma$ should be large depending on $s$. In this way we find a continuous function $\sigma: s \rightarrow \mathbf{R}_{+}$ such that $e^{i t(k(\sigma(s))+h(s))}$ converges to $\beta_{t}$. This completes the proof of (2).

We note the following easy implication; we could not prove the converse.

Proposition 9. Let $A$ be a separable $C^{*}$-algebra and I an ideal of $A$. Let $\alpha$ be an asymptotically inner flow on $A$. Then it follows that $\alpha$ leaves I invariant, the restriction $\alpha \mid I$ is asymptotically inner, and so is the quotient $\dot{\alpha} \mid A / I$. 
Proof. Let $h: \mathbf{R}_{+} \rightarrow A_{s a}$ be a continuous function such that $\alpha_{t}$ is the limit of $\operatorname{Ad} e^{i t h(s)}$ as $s \rightarrow \infty$. If $Q$ denotes the quotient map from $A$ onto $A / I$, then Ad $e^{i t Q(h(s))}$ converges to $\dot{\alpha}_{t}$ on $A / I$. Hence $\dot{\alpha}$ is asymptotically inner.

For $x \in D\left(\delta_{\alpha}\right) \cap I=D\left(\delta_{\alpha \mid I}\right)$, let $x(s)=(1+\operatorname{ad} i h(s))^{-1}\left(1+\delta_{\alpha}\right)(x)$. It then follows that $x(s) \in I,\|x(s)-x\| \rightarrow 0$, and $\left\|\operatorname{ad} i h(s)(x(s))-\delta_{\alpha}(x)\right\| \rightarrow 0$, i.e., the graph limit of ad $i h(s) \mid I$ is $\delta_{\alpha} \mid D\left(\delta_{\alpha}\right) \cap I$, which is equivalent to saying that $\max _{|t| \leq 1}\left\|\operatorname{Ad} e^{i t h(s)}(x)-\alpha_{t}(x)\right\| \rightarrow 0$ as $s \rightarrow \infty$ for $x \in I$. We will replace $h$ by a function $h^{\prime}$ of $\mathbf{R}_{+}$into $I_{s a}$ such that $\|$ ad $h(s)(x(s))-\operatorname{ad} i h^{\prime}(s)(x(s)) \| \rightarrow 0$.

For each $n \in \mathbf{N}$ let $M(n)=\max \{\|h(s)\| \mid 0 \leq s \leq n\}$, which we may suppose is positive.

Since $D\left(\delta_{\alpha}\right) \cap I$ is a separable Banach $*$-algebra, let $\left(\mathcal{F}_{n}\right)$ be an increasing sequence of finite subsets of $D\left(\delta_{\alpha}\right) \cap I$ such that $\mathcal{F}_{n}^{*}=\mathcal{F}_{n}$ and $\bigcup_{n} \mathcal{F}_{n}$ is dense in $D\left(\delta_{\alpha}\right) \cap I$. Note that then $\bigcup_{n} \mathcal{F}_{n}$ is dense in $I$ too.

Let $e_{n} \in I$ be such that $0 \leq e_{n} \leq 1,\left\|\left(1-e_{n}\right) x(s)\right\|<(n M(n))^{-1}$, and $\left\|\left(1-e_{n}\right) h(s) x(s)\right\|<n^{-1}$ for all $x \in \mathcal{F}_{n}$ and $s \in[0, n]$. We define a continuous function $e: \mathbf{R}_{+} \rightarrow I_{s a}$ by

$$
e(s)=(n-s) e_{n}+(s-n+1) e_{n+1}, \quad s \in[n-1, n],
$$

where $n=1,2, \ldots$. Then if $s \in[n-1, n]$, we get that

$$
\|(1-e(s))(x(s))\|<\frac{1}{n M(n)}
$$

and

$$
\|(1-e(s)) h(s) x(s)\|<\frac{1}{n}
$$

for all $x \in \mathcal{F}_{n}$ and $s \in[0, n]$. Let $h^{\prime}(s)=e(s) h(s) e(s) \in I$. Then, $h^{\prime}$ is a continuous function of $\mathbf{R}_{+}$into $I_{s a}$. By computation, we get that $\left\|\operatorname{ad} i h^{\prime}(s)(x(s))-\operatorname{ad} i h(s)(x(s))\right\|<4 / n$ for $x \in \mathcal{F}_{n}$ and $s \in[0, n]$ because $\left\|\left[h^{\prime}(s), x(s)\right]-[h(s), x(s)]\right\|$ is dominated by

$$
\begin{aligned}
& \|h(s)\|\|(e(s)-1) x(s)\|+\|x(s)(e(s)-1)\|\|h(s)\| \\
& +\|(e(s)-1) h(s) x(s))\|+\| x(s) h(s)(e(s)-1) \| .
\end{aligned}
$$

Thus the graph limit of ad $i h^{\prime}(s)$ as $s \rightarrow \infty$ is $\delta_{\alpha} \mid D\left(\delta_{\alpha}\right) \cap I$. This concludes the proof.

For a flow $\alpha$ we define the Connes spectrum $\mathbf{R}(\alpha)$ as a closed subgroup of $\mathbf{R}$ (see [14] for details). In the following result we actually show that the flow $\alpha$ has the following property: For any non-empty open set $O \subset \mathbf{R}$ the spectral subspace $A^{\alpha}(O)$ has a central sequence $\left(x_{n}\right)$ such that $\left\|x_{n}\right\|=1$ and $\lim _{n \rightarrow \infty}\left\|x_{n} y\right\|=\|y\|, y \in A$, which insures that $\mathbf{R}(\alpha)=\mathbf{R}$.

Theorem 10. Let $A$ be a separable $C^{*}$-algebra. Then there is an asymptotically inner flow $\alpha$ on $A$ such that the Connes spectrum $\mathbf{R}(\alpha)$ of $\alpha$ is full if and only if $A$ is antiliminary. 
Proof. This is only a slight modification of Theorem 1.3 of [8].

Suppose that there is such a flow $\alpha$ on $A$. Let $I$ be the largest ideal of $A$ such that $I$ is of type $I$ and suppose that $I \neq 0$. Then, since $\alpha$ leaves an ideal of $I$ invariant, it follows that $\alpha \mid I$ is universally weakly inner and that $\mathbf{R}(\alpha \mid I)=\{0\}$. Thus we get that $\mathbf{R}(\alpha)=\{0\}$ (since $\mathbf{R}(\alpha) \subset \mathbf{R}(\alpha \mid I)$ ), which is a contradiction. Hence $I$ must be zero, i.e., $A$ must be antiliminary.

Suppose that $A$ is antiliminary. There are a countable family $\left\{\pi_{i}\right\}$ of irreducible representations of $A$ such that $\bigcap_{i} \operatorname{ker} \pi_{i}=\{0\}$ and $\pi_{i}(A) \cap K\left(\mathcal{H}_{\pi_{i}}\right)=$ $\{0\}$, where $K\left(\mathcal{H}_{\pi}\right)$ denotes the compact operators on the Hilbert space $\mathcal{H}_{\pi_{i}}$.

In the proof of 1.3 of [8] we worked with just one of such representations, say $\pi$, and constructed a bounded central sequence $\left(h_{n}\right)$ in $A_{s a}$ such that the flow $\alpha$ is defined as the limit of $\operatorname{Ad} e^{i t H_{n}}$ and is covariant in $\pi$ with the induced flow on $\pi(A)$ having the desired properties, where $H_{n}=h_{1}+h_{2}+\cdots+h_{n}$. (More precisely we also construct a bounded central sequence $\left(b_{n}\right)$ such that various subsequences of $\left(b_{n}\right)$ would produce a sequence $\left(x_{n}\right)$ as stated before this theorem).

What we have to do now is to work with the direct sum $\pi_{1} \oplus \pi_{2} \oplus \cdots \oplus \pi_{n}$ at the $n$ 'th step of induction. The main tools we used in the proof are a version of Haagerup's result (Lemma 4.2 of [12]) and Kadison's transitivity, which are both available for finite direct sums of irreducible representations of the above type. Thus we can complete the proof in much the same way as in [8].

The flow $\alpha$ obtained this way is as a matter of fact asymptotically inner by Proposition $6 ;\left(h_{n}\right)$ satisfies that if $h: \mathbf{R}_{+} \rightarrow A_{s a}$ is defined by $h(s)=$ $H_{n-1}+(s-n+1) h_{n}, s \in[n-1, n]$ with $H_{0}=0$ for $n=1,2, \ldots$, then $\operatorname{Ad} e^{i t H(s)}$ converges to $\alpha_{t}$.

Given an antiliminary $\mathrm{C}^{*}$-algebra we get the existence of a non-trivial asymptotically inner flow as above, but we do not know how many cocycleconjugacy classes of such flows there are.

\section{Lifting}

Theorem 11. Let $A$ be a separable $C^{*}$-algebra and $I$ a (closed) ideal of $A$. Let $B=A / I$ be the quotient of $A$ by $I$ with $Q$ the canonical quotient map of $A$ onto $B$. If $\beta$ is an asymptotically inner flow on $B$, then there is an asymptotically inner flow $\alpha$ on $A$ such that $Q \alpha=\beta Q$ and $\alpha \mid I$ is universally weakly inner.

Proof. Let $h$ be a continuous function of $\mathbf{R}_{+}$into $B_{s a}$ such that

$$
\lim _{s \rightarrow \infty} \max _{|t| \leq 1}\left\|\beta_{t}(y)-\operatorname{Ad} e^{i t h(s)}(y)\right\| \rightarrow 0
$$

for every $y \in B$.

Let $\left(\mathcal{F}_{n}\right)$ be an increasing sequence of finite subsets of the unit ball $A_{1}$ of $A$ such that the union $\bigcup_{n} \mathcal{F}_{n}$ is dense in $A_{1}$ and let $\left(\epsilon_{n}\right)$ be a decreasing 
sequence of positive numbers such that $\sum_{n} \epsilon_{n}<\infty$. We choose an increasing sequence $\left(s_{n}\right)$ in $\mathbf{R}_{+}$such that for any $s \geq s_{n}$ and $y \in Q\left(\mathcal{F}_{n}\right)$,

$$
\max _{|t| \leq 1}\left\|\beta_{t}(y)-\operatorname{Ad} e^{i t h(s)}(y)\right\|<\epsilon_{n} .
$$

Since $I$ is a separable ideal, there is an approximate identity $\left(e_{n}\right)$ in $I$ such that $e_{n} e_{n+1}=e_{n}$ and

$$
\left\|\left[x, e_{n}\right]\right\| \rightarrow 0, \quad x \in A .
$$

It also follows that $\|Q(x)\|=\lim _{n}\left\|x\left(1-e_{n}\right)\right\|$ for any $x \in A$. We will use these facts in the arguments below.

We will find a continuous function $H$ of $\mathbf{R}_{+}$into $A_{s a}$ such that $Q\left(H\left(s_{n}\right)\right)=$ $h\left(s_{n}\right)$ and

$$
\max _{|t| \leq 1}\left\|\operatorname{Ad} e^{i t H\left(s_{n}\right)}(x)-\operatorname{Ad} e^{i t H(s)}(x)\right\|<7 \epsilon_{n}
$$

for $s \in\left[s_{n}, s_{n+1}\right]$ and $x \in \mathcal{F}_{n}$ and $H(s) e_{n}=H\left(s_{n}\right) e_{n}$ for $s \geq s_{n}$ and $n=$ $1,2, \ldots$, where $\left(e_{n}\right)$ is an approximate unit for $I$. Then it would follow that Ad $e^{i t H(s)}(x)$ converges uniformly in $t \in[-1,1]$ for any $x \in A$ and thus defines an asymptotically inner flow $\alpha$ on $A$. Since $Q\left(H\left(s_{n}\right)\right)=h\left(s_{n}\right)$, we get that $Q \alpha_{t}=\beta_{t} Q$.

Since $\delta_{\alpha}\left|\overline{e_{n} A e_{n}}=\operatorname{ad} i H\left(s_{n}\right)\right| \overline{e_{n} A e_{n}}$, we get that $\delta_{\alpha}-\operatorname{ad} i H\left(s_{n}\right)$ vanishes on $\overline{e_{n} A e_{n}}$. Hence the flow $\alpha^{\left(-H\left(s_{n}\right)\right)}$ generated by $\delta_{\alpha}-$ ad $i H\left(s_{n}\right)$ fixes each element of $\overline{e_{n} A e_{n}}$. This implies that if $\phi$ is a state of $A$ such that $\left\|\phi \mid \overline{e_{n} A e_{n}}\right\|=1$, then $\pi_{\phi}$ is covariant under $\alpha$, which is just an inner perturbation of $\alpha^{\left(-H\left(s_{n}\right)\right)}$. Since the set of states $\phi$ with the property $\left\|\phi \mid \overline{e_{n} A e_{n}}\right\|=1$ for some $n$ is dense in the states of $I$, we get that $\alpha \mid I$ is universally extendible (i.e., $t \mapsto \alpha_{t}^{* *}(x)$ is weak*-continuous for $x \in I^{* *}$ ), which is equivalent to being universally weakly inner [7]. Thus $\alpha$ has the desired properties.

Now we turn to the construction of $H: \mathbf{R}_{+} \rightarrow A_{s a}$. We fix an approximate unit $\left(e_{n}\right)$ for $I$ such that $e_{n} e_{n+1}=e_{n}$ for all $n$. We choose an $H_{1} \in A_{s a}$ such that $Q\left(H_{1}\right)=h\left(s_{1}\right)$ and set $H(s)=\left(s / s_{1}\right) H_{1}$ for $s \in\left[0, s_{1}\right]$.

Suppose that we have defined a continuous function $H:\left[0, s_{n}\right] \rightarrow A_{s a}$ such that

$$
\max _{|t| \leq 1}\left\|\operatorname{Ad} e^{i t H\left(s_{k}\right)}(x)-\operatorname{Ad} e^{i t H(s)}(x)\right\|<7 \epsilon_{k}, \quad x \in \mathcal{F}_{k}
$$

for $s \in\left[s_{k}, s_{k+1}\right]$ and $k=1,2, \ldots, n-1$ and

$$
H(s) e_{k}=H\left(s_{k}\right) e_{k}, \quad s \in\left[s_{k}, s_{k+1}\right] .
$$

We find a continuous function $K:\left[s_{n}, s_{n+1}\right] \rightarrow A_{s a}$ such that $K\left(s_{n}\right)=$ $H\left(s_{n}\right)$ and $Q(K(s))=h(s), s \in\left[s_{k}, s_{k+1}\right]$. Since $\max _{|t| \leq 1} \| \operatorname{Ad} e^{i t h\left(s_{n}\right)}(y)-$ $\operatorname{Ad} e^{i t h(s)}(y) \|<2 \epsilon_{n}$ for $s \in\left[s_{n}, s_{n+1}\right]$ and $y \in Q\left(\mathcal{F}_{n}\right)$, we get that

$$
\left\|Q\left(\operatorname{Ad} e^{i t K\left(s_{n}\right)}(x)-\operatorname{Ad} e^{i t K(s)}(x)\right)\right\|<2 \epsilon_{n}, \quad t \in[-1,1], x \in \mathcal{F}_{n}
$$

for $s \in\left[s_{n}, s_{n+1}\right]$. Hence there is an $e=e_{m} \in I$ for some $m \geq n$ such that 


$$
\left\|(1-e)\left(\operatorname{Ad} e^{i t K(s)} \operatorname{Ad} e^{-i t K\left(s_{n}\right)}(x)-x\right)\right\|<2 \epsilon_{n}, \quad t \in[-1,1], x \in \mathcal{F}_{n}
$$

for $s \in\left[s_{n}, s_{n+1}\right]$.

Let $\gamma$ denote the flow $t \mapsto \operatorname{Ad} e^{i t K\left(s_{n}\right)}=\operatorname{Ad} e^{i t H\left(s_{n}\right)}$ on $A$. Then $u^{(s)}: t \mapsto$ $e^{i t K(s)} e^{-i t K\left(s_{n}\right)}$ is a $\gamma$-cocycle. We set $W(s)=K(s)-K\left(s_{n}\right)$ and note that

$$
u_{t}^{(s)}=\sum_{n=0}^{\infty} \int \cdots \int_{\Gamma_{n}(t)} d t_{1} d t_{2} \cdots d t_{n} i^{n} \gamma_{t_{1}}(W(s)) \gamma_{t_{2}}(W(s)) \cdots \gamma_{t_{n}}(W(s))
$$

where $\Gamma_{n}(t)$ means $0 \leq t_{1} \leq t_{2} \leq \cdots \leq t_{n} \leq t$ if $t \geq 0$ and similar inequalities otherwise. Let $M=\max _{s \in\left[s_{n}, s_{n+1}\right]}\|W(s)\|$ and let $N \in \mathbf{N}$ be such that

$$
\sum_{n=N+1}^{\infty} \frac{M^{n}}{n !}<\epsilon_{n} / 8
$$

We will choose a $\delta>0$ very small below. We find a finite sequence $\left(\sigma_{i}\right)_{i=0}^{L}$ in $\left[s_{n}, s_{n+1}\right]$ such that $s_{n}=\sigma_{0}<\sigma_{2}<\cdots<\sigma_{L}=s_{n+1}$ and $\| K\left(\sigma_{i}\right)-$ $K\left(\sigma_{i-1}\right) \|<\delta$ for $i=1,2, \ldots, L$. We find a finite sequence $\left(p_{i}\right)_{i=0}^{K}$ in $I$ such that $0 \leq p_{i} \leq 1, p_{0} e=e, p_{i} p_{i+1}=p_{i}$, and

$$
\left\|\left[p_{i}, K(s)\right]\right\|,\left\|\left[p_{i}, x\right]\right\|, \quad s \in\left[s_{n}, s_{n+1}\right], x \in \mathcal{F}_{n}
$$

are all very small.

For $i=1,2, \ldots, L$ we define

$W_{i}=\sum_{k=1}^{i-1}\left(p_{k}-p_{k-1}\right)^{1 / 2} W\left(\sigma_{k}\right)\left(p_{k}-p_{k-1}\right)^{1 / 2}+\left(1-p_{i-1}\right)^{1 / 2} W\left(\sigma_{i}\right)\left(1-p_{i-1}\right)^{1 / 2}$.

Since $\left\|W\left(\sigma_{i}\right)-W\left(\sigma_{i-1}\right)\right\|<\delta$ and $\left(p_{k}-p_{k-1}\right)^{1 / 2}$ and $W(\sigma)$ almost commute, it follows that $\left\|W_{i}-W_{i-1}\right\|$ is at most of the order of $\delta$. It also follows that $W_{i} e_{n}=0$ as $p_{0} e_{n}=e_{n}$.

For $i=1,2, \ldots, L$ and $t \in \mathbf{R}$ we define $u_{t}^{(i)}=e^{i t\left(K\left(s_{n}\right)+W_{i}\right)} e^{-i t K\left(s_{n}\right)}$, which is a $\gamma$-cocycle. Since $\left\|W_{i}\right\| \leq M$, the norm difference of $u_{t}^{(i)}$ and

$$
\sum_{n=0}^{N} \int \cdots \int_{\Gamma_{n}(t)} d t_{1} \cdots d t_{n} i^{n} \gamma_{t_{1}}\left(W_{i}\right) \gamma_{t_{2}}\left(W_{i}\right) \cdots \gamma_{t_{n}}\left(W_{i}\right)
$$

is smaller than $\epsilon_{n} / 8$ for $t \in[-1,1]$.

Let $j=1,2, \ldots, i-1$. We shall identify $\left(p_{j}-p_{j-1}\right) u_{t}^{i}$. Since $\|\left(p_{j}-\right.$ $\left.p_{j-1}\right)\left(W_{i}-W\left(\sigma_{j}\right)\right) \|<\delta$ and $\left(p_{k}-p_{k-1}\right)^{1 / 2}$ is almost invariant under $\gamma$ and are almost central as we have assumed, we can derive that

$$
\left\|\left(p_{j}-p_{j-1}\right)\left(\gamma_{t_{1}}\left(W_{i}\right) \cdots \gamma_{t_{n}}\left(W_{i}\right)-\gamma_{t_{1}}\left(W\left(\sigma_{j}\right)\right) \cdots \gamma_{t_{n}}\left(W\left(\sigma_{j}\right)\right)\right)\right\|
$$

is of the order of $\delta n M^{n-1}$, where we used that $\left|t_{k}\right| \leq 1$. Thus we get that 


$$
\left\|\left(p_{j}-p_{j-1}\right)\left(u_{t}^{(i)}-e^{i t\left(K\left(s_{n}\right)+W\left(\sigma_{j}\right)\right)} e^{-i t K\left(s_{n}\right)}\right)\right\|<C \delta\left(e^{M}-1\right)+\epsilon_{n} / 4,
$$

for $t \in[-1,1]$, where $C$ is a constant depending on $N$ and $M$ but can be made arbitrarily close to 1 by taking $p_{k}$ 's so that they almost commute with $K(s)$ 's. So our choice of $\delta$ must be made such that $C \delta\left(e^{M}-1\right)$ is smaller than $\epsilon_{n} / 4$. In the same way we get that

$$
\left\|\left(1-p_{i-1}\right)\left(u_{t}^{(i)}-e^{i t\left(K\left(s_{n}\right)+W\left(\sigma_{i}\right)\right)} e^{-i t K\left(s_{n}\right)}\right)\right\|<\epsilon_{n} / 2
$$

and

$$
\left\|\left(1-p_{0}\right)\left(u_{t}^{(i)}-1\right)\right\|<\epsilon_{n} / 2 .
$$

Since $1-e$ dominates $p_{j}-p_{j-1}$ etc. and $p_{k}$ 's almost commute with $x \in \mathcal{F}_{n}$ and $K(s)$, we get that

$$
\left\|\left(p_{j}-p_{j-1}\right)\left(u_{t}^{(i)} x\left(u_{t}^{(i)}\right)^{*}-x\right)\right\|<2 \epsilon_{n}+2 \cdot \epsilon_{n} / 2=3 \epsilon_{n},
$$

for $t \in[-1,1]$. Together with similar estimates we can show that

$$
\left\|\operatorname{Ad} u_{t}^{(i)}(x)-x\right\|<6 \epsilon_{n}, \quad x \in \mathcal{F}_{n}, \quad t \in[-1,1],
$$

where we use that $\left(p_{j}-p_{j-1}\right)\left(p_{k}-p_{k-1}\right)=0$ for $|j-k|>1$ etc. and that $\left[p_{j}-p_{j-1}, u_{t}^{(i)} x\left(u_{t}^{(i)}\right)^{*}-x\right] \approx 0$ as closely as we wish.

Thus we have constructed $W_{i}, i=1,2, \cdots L$ such that $\left\|W_{i}-W_{i-1}\right\|$ is of the order of $\delta, Q\left(W_{i}\right)=h\left(\sigma_{i}\right)-h\left(s_{n}\right)$, and

$$
\left\|\operatorname{Ad} e^{i t\left(K\left(s_{n}\right)+W_{i}\right)}(x)-\operatorname{Ad} e^{i t K\left(s_{n}\right)}(x)\right\|<6 \epsilon_{n}, \quad x \in \mathcal{F}_{n}, t \in[-1,1] .
$$

We define a continuous $H:\left[s_{n}, s_{n+1}\right] \rightarrow A_{s a}$ as follows: if $s \in\left[\sigma_{j-1}, \sigma_{j}\right]$, then

$$
H(s)=\frac{\sigma_{j}-s}{\sigma_{j}-\sigma_{j-1}}\left(H\left(s_{n}\right)+W_{j-1}\right)+\frac{s-\sigma_{j-1}}{\sigma_{j}-\sigma_{j-1}}\left(H\left(s_{n}\right)+W_{j}\right) .
$$

Since $\left\|H(s)-\left(H\left(s_{n}\right)+W_{j}\right)\right\|<\delta$ for such $s$, we have that $\| e^{i t H(s)}-$ $e^{i t\left(H\left(s_{n}\right)+W_{j}\right)} \|<\delta$ for $t \in[-1,1]$. Thus we have that

$$
\left\|\operatorname{Ad} e^{i t H\left(s_{n}\right)}(x)-\operatorname{Ad} e^{i t H(s)}(x)\right\|<6 \epsilon_{n}+2 \delta<7 \epsilon_{n}, \quad x \in \mathcal{F}_{n}, \quad t \in[-1,1] .
$$

We also note that $H(s) e_{n}=H\left(s_{n}\right) e_{n}$ for $s \in\left[s_{n}, s_{n+1}\right]$ and that $Q\left(H\left(s_{n+1}\right)\right)=$ $Q\left(H\left(s_{n}\right)+W\left(s_{n+1}\right)\right)=h\left(s_{n+1}\right)$. This completes the proof.

Proposition 12. Let $A$ be a separable $C^{*}$-algebra and $I$ an ideal of $A$. Let $\beta$ be an asymptotically inner automorphism of the quotient $B=A / I$ in the sense that there is a continuous map $u: \mathbf{R}_{+} \rightarrow \mathcal{U}(B)$ such that $\beta=\lim _{s \rightarrow \infty} \operatorname{Ad} u(s)$. Moreover suppose that $u$ satisfies that $u(0) \in Q(\mathcal{U}(A))$, where $Q$ is the quotient map of $A$ onto $B$. Then there is an asymptotically inner automorphism $\alpha$ of $A$ such that $Q \alpha=\beta Q$ and $\alpha \mid I$ is universally weakly inner. 
Proof. Let $\left(\mathcal{F}_{n}\right)$ be an increasing sequence of finite subsets of the unit ball $A_{1}$ such that $\bigcup_{n} A_{n}$ is dense in $A_{1}$ and let $\left(\epsilon_{n}\right)$ be a decreasing sequence of positive numbers such that $\sum_{n} \epsilon_{n}<\infty$. Let $u: \mathbf{R}_{+} \rightarrow \mathcal{U}(B)$ be as in the statement. We note that $\beta^{-1}=\lim _{s \rightarrow \infty} \operatorname{Ad} u(s)^{*}$ since $\|\beta(y)-\operatorname{Ad} u(s)(y)\|=$ $\left\|\beta^{-1} \beta(y)-\operatorname{Ad} u(s)^{*} \beta(y)\right\|$.

We find an increasing sequence $\left(s_{n}\right)$ in $\mathbf{R}_{+}$such that for all $s \geq s_{n}$ and $y \in Q\left(\mathcal{F}_{n}\right)$,

$$
\begin{aligned}
\|\beta(y)-\operatorname{Ad} u(s)(y)\| & <\epsilon_{n}, \\
\left\|\beta^{-1}(y)-\operatorname{Ad} u(s)^{*}(y)\right\| & <\epsilon_{n} .
\end{aligned}
$$

We will define a continuous map $U: \mathbf{R}_{+} \rightarrow \mathcal{U}(A)$ such that $Q\left(U\left(s_{n}\right)\right)=$ $u\left(s_{n}\right)$ for all $n$ and

$$
\begin{aligned}
\left\|\operatorname{Ad} U(s)(x)-\operatorname{Ad} U\left(s_{n}\right)(x)\right\| & <5 \epsilon_{n}, \\
\left\|\operatorname{Ad} U(s)^{*}(x)-\operatorname{Ad} U\left(s_{n}\right)^{*}(x)\right\| & <5 \epsilon_{n}
\end{aligned}
$$

for all $x \in \mathcal{F}_{n}$ and $s \in\left[s_{n}, s_{n+1}\right]$ and for all $n$.

Since $\sum_{n} \epsilon_{n}<\infty$, this implies that $(\operatorname{Ad} U(s)(x))$ is a Cauchy sequence for $x \in \bigcup_{n} \mathcal{F}_{n}$. Hence we can define an endomorphism $\alpha$ of $A$ by $\alpha(x)=$ $\lim _{s \rightarrow \infty} \operatorname{Ad} U(s)(x)$. Since we can also define an endomorphism $\gamma$ of $A$ by $\gamma(x)=\lim _{s \rightarrow \infty} \operatorname{Ad} U(s)^{*}(x)$ such that $\alpha \gamma=$ id $=\gamma \alpha$, we get that $\alpha$ is an automorphism. Since $Q\left(U\left(s_{n}\right)\right)=u\left(s_{n}\right)$, we also have that $Q \alpha=$ $\lim _{n \rightarrow \infty} \operatorname{Ad} u\left(s_{n}\right) Q=\beta Q$.

We will require the map $U: \mathbf{R}_{+} \rightarrow \mathcal{U}(A)$ to satisfy an additional condition as follows. There is an approximate unit $\left(e_{n}\right)$ in $I$ such that $e_{n} e_{n+1}=e_{n}$ and $U(s) U\left(s_{n}\right)^{*} e_{n}=e_{n}$ for $s \geq s_{n}$. This implies that $\alpha \operatorname{Ad} U\left(s_{n}\right)^{*}\left|I_{n}=\mathrm{id}\right| I_{n}$, where $I_{n}=\overline{e_{n} I e_{n}}=\overline{e_{n} A e_{n}}$. Hence if $\phi$ is a state of $A$ such that $\left\|\phi \mid I_{n}\right\|=1$, then $\phi \alpha \operatorname{Ad} U\left(s_{n}\right)^{*}=\phi$, i.e., $\pi_{\phi}$ is covariant under $\alpha$. Since $\bigcup_{n} I_{n}$ is dense in $I$, we get that if $\phi$ is a state of $I$, then $\pi_{\phi}$ is covariant under $\alpha$. Hence we can conclude that $\alpha \mid I$ is universally weakly inner [7].

Now we turn to the construction of such $U: \mathbf{R}_{+} \rightarrow \mathcal{U}(A)$.

We have specified $u: \mathbf{R}_{+} \rightarrow \mathcal{U}(B)$ and $\left(s_{n}\right)$, such that

$$
\begin{aligned}
\left\|\operatorname{Ad} u(s)(y)-\operatorname{Ad} u\left(s_{n}\right)(y)\right\| & <2 \epsilon_{n}, \\
\left\|\operatorname{Ad} u(s)^{*}(y)-\operatorname{Ad} u\left(s_{n}\right)^{*}(y)\right\| & <2 \epsilon_{n}
\end{aligned}
$$

for all $y \in Q\left(\mathcal{F}_{n}\right)$ and $s \in\left[s_{n}, s_{n+1}\right]$ and all $n=1,2, \ldots$. We also specify an approximate unit $\left(e_{n}\right)$ for $I$ such that $e_{n} e_{n+1}=e_{n}$ for all $n$.

Let $s_{0}=0$ and we choose a continuous map $U:\left[s_{0}, s_{1}\right] \rightarrow \mathcal{U}(A)$ such that $Q(U(s))=u(s)$ for $s=s_{0}$ and $s=s_{1}$ (or for all $s \in\left[s_{0}, s_{1}\right]$ ). This is possible by the assumption.

Suppose that we have defined $U:\left[s_{0}, s_{n}\right] \rightarrow \mathcal{U}(A)$ as required, i.e., we have that $Q\left(U\left(s_{k}\right)\right)=u\left(s_{k}\right), U(s) U\left(s_{k}\right)^{*} e_{k}=e_{k}$ for $s \in\left[s_{k}, s_{k+1}\right]$, and

$$
\begin{aligned}
\left\|\operatorname{Ad} U(s)(x)-\operatorname{Ad} U\left(s_{n}\right)(x)\right\| & <5 \epsilon_{k}, \\
\left\|\operatorname{Ad} U(s)^{*}(x)-\operatorname{Ad} U\left(s_{n}\right)^{*}(x)\right\| & <5 \epsilon_{k}
\end{aligned}
$$


for all $x \in \mathcal{F}_{k}$ and $s \in\left[s_{k}, s_{k+1}\right]$ and for $k=1,2, \ldots, n-1$.

We choose a continuous $V:\left[s_{n}, s_{n+1}\right] \rightarrow \mathcal{U}(A)$ such that $V\left(s_{n}\right)=U\left(s_{n}\right)$ and $Q(V(s))=u(s), s \in\left[s_{n}, s_{n+1}\right]$. Since

$$
\begin{gathered}
\left\|Q\left(\operatorname{Ad} V(s)(x)-\operatorname{Ad} V\left(s_{n}\right)(x)\right)\right\|<2 \epsilon_{k}, \\
\left\|Q\left(\operatorname{Ad} V(s)^{*}(x)-\operatorname{Ad} V\left(s_{n}\right)^{*}(x)\right)\right\|<2 \epsilon_{k}
\end{gathered}
$$

for $x \in \mathcal{F}_{n}$ and $s \in\left[s_{n}, s_{n+1}\right]$, we find an $e=e_{m} \in I$ for some $m \geq n$ such that

$$
\begin{gathered}
\left\|(1-e)\left(\operatorname{Ad} V(s)(x)-\operatorname{Ad} V\left(s_{n}\right)(x)\right)\right\|<2 \epsilon_{k}, \\
\left\|(1-e)\left(\operatorname{Ad} V(s)^{*}(x)-\operatorname{Ad} V\left(s_{n}\right)^{*}(x)\right)\right\|<2 \epsilon_{k}
\end{gathered}
$$

for $x \in \mathcal{F}_{n}$ and $s \in\left[s_{n}, s_{n+1}\right]$.

Let $\delta>0$, which will be chosen later to be a sufficiently small constant. Let $\left(t_{i}\right)_{i=0}^{N}$ be a sequence in $\left[s_{n}, s_{n+1}\right]$ such that $s_{n}=t_{0}<t_{1}<t_{2}<\cdots<$ $t_{N}=s_{n+1}$ and

$$
\left\|V\left(t_{i}\right)-V\left(t_{i-1}\right)\right\|<\delta
$$

for $i=1,2, \ldots, N$. We find a sequence $\left(f_{i}\right)_{i=0}^{N}$ in $I$ such that $0 \leq f_{i} \leq 1$, $e f_{0}=e, f_{i} f_{i+1}=f_{i}$, and

$$
\left\|\left[f_{i}, V\left(t_{j}\right)\right]\right\| \approx 0, \quad\left\|\left[f_{i}, x\right]\right\| \approx 0
$$

for all $i, j$ and $x \in \mathcal{F}_{n}$. We define a sequence $\left(W_{i}\right)_{i=0}^{N}$ in $\tilde{A}$ by $W_{0}=1$ and

$$
W_{i}=f_{0}+\sum_{j=1}^{i-1} V\left(t_{j}\right) V\left(t_{0}\right)^{*}\left(f_{j}-f_{j-1}\right)+V\left(t_{i}\right) V\left(t_{0}\right)^{*}\left(1-f_{i-1}\right)
$$

for $i=1,2, \ldots, N$. If $0<j<i$, then we have that

$$
\begin{aligned}
W_{i}\left(f_{j}-f_{j-1}\right)= & V\left(t_{j-1}\right) V\left(t_{0}\right)^{*}\left(f_{j-1}-f_{j-2}\right)\left(f_{j}-f_{j-1}\right) \\
& +V\left(t_{j}\right) V\left(t_{0}\right)^{*}\left(f_{j}-f_{j-1}\right)^{2} \\
& +V\left(t_{j+1}\right) V\left(t_{0}\right)^{*}\left(f_{j+1}-f_{j}\right)\left(f_{j}-f_{j-1}\right) \\
= & V\left(t_{j-1}\right) V\left(t_{0}\right)^{*}\left(f_{j-1}-f_{j-1}^{2}\right) \\
& +V\left(t_{j}\right) V\left(t_{0}\right)^{*}\left(f_{j}^{2}+f_{j-1}^{2}-2 f_{j-1}\right) \\
& +V\left(t_{j+1}\right) V\left(t_{0}\right)^{*}\left(f_{j}-f_{j}^{2}\right) .
\end{aligned}
$$

By replacing $V\left(t_{j \pm 1}\right) V\left(t_{0}\right)^{*}$ by $V\left(t_{j}\right) V\left(t_{0}\right)^{*}$, we get that

$$
\left\|\left(W_{i}-V\left(t_{j}\right) V\left(t_{0}\right)^{*}\right)\left(f_{j}-f_{j-1}\right)\right\|<\delta .
$$

Moreover we have that $\left\|\left(W_{i}-1\right) f_{0}\right\|<\delta$ and $\left\|\left(W_{i}-V\left(t_{i}\right) V\left(t_{0}\right)^{*}\right)\left(1-f_{i-1}\right)\right\|<$ $\delta$. Assuming that $\left\|\left[V\left(t_{j}\right) V\left(t_{0}\right)^{*}, f_{k}-f_{k-1}\right]\right\| \approx 0$, we get that $\|\left(W_{i}^{*} W_{i}-\right.$ 1) $\left(f_{j}-f_{j-1}\right) \|<2 \delta$ etc., where we have ignored an error of $\delta^{2}$ (which may 
result if $\left.\left\|W_{i}\right\|>1\right)$. Hence, by taking the summation over $j$ and noting that $\left(f_{j}-f_{j-1}\right)\left(f_{k}-f_{k-1}\right)=0$ for $j>k+1$, we can conclude that

$$
\left\|W_{i}^{*} W_{i}-1\right\|<4 \delta .
$$

In the same way we get that $\left\|W_{i} W_{i}^{*}-1\right\|<4 \delta$, i.e., $W_{i}$ is close to a unitary.

Note also that $\left\|W\left(t_{i}\right)-W\left(t_{i-1}\right)\right\|<\delta$, which follows from

$$
W_{i}-W_{i-1}=-V\left(t_{i-1}\right) V\left(t_{0}\right)^{*}\left(1-f_{i-1}\right)+V\left(t_{i}\right) V\left(t_{0}\right)^{*}\left(1-f_{i-1}\right) .
$$

We will claim that $\Delta_{i} \equiv W_{i} U\left(s_{n}\right) x U\left(s_{n}\right)^{*} W_{i}^{*}-U\left(s_{n}\right) x U\left(s_{n}\right)^{*} \approx 0$ for $x \in \mathcal{F}_{n}$. Since $V\left(t_{0}\right)=U\left(s_{n}\right)$, this follows because if $0<j<i$,

$$
\Delta_{i}\left(f_{j}-f_{j-1}\right) \approx\left(V\left(t_{j}\right) x V\left(t_{j}\right)^{*}-V\left(t_{0}\right) x V\left(t_{0}\right)^{*}\right)(1-e)\left(f_{j}-f_{j-1}\right)
$$

with an error of at most $2 \delta$ assuming that $\left\|\left[V\left(t_{k}\right), f_{j}-f_{j-1}\right]\right\| \approx 0$ and $\|\left[x, f_{j}-\right.$ $\left.f_{j-1}\right] \| \approx 0$. Thus we get that

$$
\left\|\left(W_{i} \operatorname{Ad} U\left(s_{n}\right)(x) W_{i}^{*}-\operatorname{Ad} U\left(s_{n}\right)(x)\right)\left(f_{j}-f_{j-1}\right)\right\|<2 \epsilon_{n}+2 \delta .
$$

Together with similar inequalities with $f_{0}$ and $1-f_{i-1}$ in place of $f_{j}-f_{j-1}$, we get that

$$
\left\|W_{i} \operatorname{Ad} U\left(s_{n}\right)(x) W_{i}^{*}-\operatorname{Ad} U\left(s_{n}\right)(x)\right\|<4\left(\epsilon_{n}+\delta\right) .
$$

Define a continuous function $W:\left[s_{n}, s_{n+1}\right] \rightarrow \mathcal{U}(A)$ by

$$
W(t)=\frac{t_{i}-t}{t_{i}-t_{i-1}} W_{i-1}+\frac{t-t_{i-1}}{t_{i}-t_{i-1}} W_{i}, \quad t \in\left[t_{i-1}, t_{i}\right]
$$

for $i=1,2, \ldots, N$. Since $\left\|W(t)-W_{i}\right\|<\delta$ for $t \in\left[t_{i-1}, t_{i}\right]$, we get that

$$
\left\|W(t) \operatorname{Ad} U\left(s_{n}\right)(x) W(t)^{*}-\operatorname{Ad} U\left(s_{n}\right)(x)\right\|<4 \epsilon_{n}+6 \delta .
$$

We let $U(s)$ be the unitary obtained from the polar decomposition of $W(s) U\left(s_{n}\right)$ for $s \in\left[s_{n}, s_{n+1}\right]$. Then by choosing $\delta>0$ sufficiently small (or roughly $12 \delta<\epsilon_{n}$; see below), we get that

$$
\left\|\operatorname{Ad} U(s)(x)-\operatorname{Ad} U\left(s_{n}\right)(x)\right\|<5 \epsilon_{n}, \quad x \in \mathcal{F}_{n} .
$$

Similarly we can also require that

$$
\left\|\operatorname{Ad} U(s)^{*}(x)-\operatorname{Ad} U\left(s_{n}\right)^{*}(x)\right\|<5 \epsilon_{n}, \quad x \in \mathcal{F}_{n} .
$$

By the construction we also have that $U(s) U\left(s_{n}\right)^{*} e_{n}=e_{n}$. This concludes the proof.

Lemma 13. Let $\delta \in(0,1 / 2)$ and let $W \in \tilde{A}=A+\mathbf{C} 1$ be such that $\| W W^{*}-$ $1 \|<\delta$ and $\left\|W^{*} W-1\right\|<\delta$. If $U$ denotes the unitary obtained from the polar decomposition of $W$, then $\|W\|<2$ and $\|U-W\|<2 \delta$. Hence for $x \in A$ with $\|x\| \leq 1$, it follows that $\left\|U x U^{*}-W x W^{*}\right\|<6 \delta$.

The proof of this lemma is standard. 


\section{References}

1. O. Bratteli and A. Kishimoto, Generation of semigroups and two-dimensional quantum lattice systems, J. Funct. Analysis 35 (1980), 344-368.

2. O. Bratteli and D.W. Robinson, Operator Algebras and Quantum Statistical Mechanics, I, Springer, 1979.

3. O. Bratteli and D.W. Robinson, Operator Algebras and Quantum Statistical Mechanics, II, Springer, 1981.

4. G.A. Elliott, Universally weakly inner one-parameter automorphism groups of separable C ${ }^{*}$-algebras, Math. Scand. 45 (1979), 149-146.

5. E. Kirchberg and N.C. Phillips, Embedding of exact $\mathrm{C}^{*}$-algebras in the Cuntz algebra $\mathcal{O}_{2}$, J. reine angew. Math. 525 (2000), 17-53.

6. E. Kirchberg and N.C. Phillips, Embedding of continuous fields of $\mathrm{C}^{*}$-algebras in the Cuntz algebra $\mathcal{O}_{2}$, J. reine angew. Math. 525 (2000), 55-94.

7. A. Kishimoto, Universally weakly inner one-parameter automorphism groups of $\mathrm{C}^{*}$-algebras, Yokohama Math. J. 30 (1982), 141-149.

8. A. Kishimoto, Approximately inner flows on separable $\mathrm{C}^{*}$-algebras, Rev. Math. Phys. 14 (2002), 649-673.

9. A. Kishimoto, Rohlin flows on the Cuntz algebra $\mathcal{O}_{2}$, Internat. J. Math. 13 (2002), 1065-1094.

10. A. Kishimoto, Rohlin property for flows, Contemporary Math. 335 (2003), 195207.

11. A. Kishimoto, Central sequence algebras of a purely infinite simple $\mathrm{C}^{*}$-algebra, Canad. J. Math. 56 (2004), 1237-1258.

12. A. Kishimoto, N. Ozawa, and S. Sakai, Homogeneity of the pure state space of a separable $\mathrm{C}^{*}$-algebra, Canad. Math. Bull. 46 (2003), 365-372.

13. D. Olesen and G.K. Pedersen, Groups of automorphisms with spectrum condition and the lifting problem, Commun. Math. Phys. 51 (1976), 85-95.

14. G.K. Pedersen, $C^{*}$-algebras and their automorphism groups, Academic Press, 1979.

15. S. Sakai, Operator Algebras in Dynamical Systems, Cambridge Univ. Press, 1991. 